\title{
Environmental Valuation under Dynamic Consumer Behavior
}

\author{
Jinhua Zhao and Catherine L. Kling \\ Working Paper 02-WP 292 \\ January 2002
}

\section{Center for Agricultural and Rural Development \\ lowa State University \\ Ames, lowa 50011-1070 \\ www.card.iastate.edu}

Jinhua Zhao is assistant professor of economics, lowa State University. Catherine Kling is professor of economics and head of the Resource and Environmental Policy Division at CARD.

This publication is available online on the CARD website: www.card.iastate.edu. Permission is granted to reproduce this information with appropriate attribution to the author and the Center for Agricultural and Rural Development, lowa State University, Ames, lowa 50011-1070.

For questions or comments about the contents of this paper, please contact Jinhua Zhao, 166D Heady Hall, lowa State University, Ames, IA 50011-1070; Ph: 515-294-5857; Fax: 515-294-0221; E-mail: jzhao@iastate.edu. 


\begin{abstract}
The paper presents two simple models of dynamic consumer behavior, both taking into consideration the implications for welfare measurement when agents can delay transactions while obtaining additional information. One model studies the effect when a purchased good is non-perishable and can be consumed in the future, while the other model introduces a perishable good, implying that the quantity of consumption can vary in each period. Even in the case of the perishable item, the availability of information at the time of the consumption decision has important implications for welfare measurement. Agents who must make a decision at the present but know that additional information will be available later may change their income allocation to take advantage of the future information. When this leads to the capture of different information sets at these different times, welfare assessment may be (but is not necessarily) inconsistent with the empirical evidence and may be inappropriate for use in policy valuation.
\end{abstract}

Key Words: intertemporal welfare measures, welfare measurement, willingness to pay. 


\section{ENVIRONMENTAL VALUATION UNDER DYNAMIC CONSUMER BEHAVIOR}

Hicksian welfare theory, the core of modern welfare analysis, forms the theoretical foundation of environmental valuation. The welfare impact of an environmental change is measured by compensating variation $(\mathrm{CV})$ or equivalent variation $(\mathrm{EV})$ or their conceptually equivalent measures: willingness to pay (WTP) and willingness to accept (WTA). While these welfare measureas are derived from a static model, consumers live in a dynamic world where they may be uncertain about the value of environmental goods, their information about goods is constantly being updated, and they may have opportunities to delay their purchase or selling decisions. In such a setting, a buyer may simultaneously form her WTP for a good as well as whether to undertake the transaction now or delay her decision, thereby opting to investigate other alternatives, consider whether the good is really "worth it" to her, or seek any other information of value in her decision.

In this paper, we argue that the application of static Hicksian welfare theory in a dynamic setting can yield incorrect welfare assessments and policy recommendations. Although there are many goods for which this scenario seems likely to hold, this issue may be particularly important in environmental valuation. Specifically, many decisions relating to the environment are inherently dynamic, with uncertain benefits and significant transaction costs associated with reversing the decisions that are made. If agents expect that more information can be gathered in the future, they may wish to delay their decision until such information is available. If they are forced to act now, they will change their WTP or WTA to incorporate compensation for their lost learning opportunity, as well as their implicit valuation of the good (i.e, expected CV or EV). Thus, static Hicksian theory, when applied to such dynamic settings, can produce predictions that (1) are inconsistent with the empirical evidence, such as the well known WTP/WTA divergence puzzle, and (2) may be inappropriate for policy assessment.

Even for decisions that are easy to reverse in the future, uncertainty and future learning may still affect the agent's demand curve. For example, deciding the number of trips to a park this year will 
not directly restrict the future visits to the park. However, future learning may affect the agent's intertemporal allocation of her lifetime income among the visits in different time periods. Then the demand curve for the current period will also be contingent on the agent's current information.

We consider two kinds of goods or services: perishable goods, which can only be consumed in the current period if purchased, and non-perishable goods, which can be consumed forever if purchased now. For perishable goods, the current consumption level does not lock in the future, so that the consumption can be freely adjusted in each period. For non-perishable goods, if the trading is irreversible, the consumption level, if chosen now, will be fixed for lengthy periods of time. Their major distinction is that the consumption level based on the current information will have long-run effects for non-perishable, but not for perishable goods. For example, if an agent is deciding how much to pay for preserving the Grand Canyon, the good of interest, i.e., the Canyon, is a nonperishable good, because if preserved, the Canyon will provide environmental amenities for a long time. If, on the other hand, the agent is asked how many trips she will make to the Canyon under certain conditions (such as the gate fee or the air quality), the good of interest, i.e., visiting the Canyon, is a perishable good. Her number of visits this year does not directly lock in her future visits.

In many cases, perishable goods are divisible and non-perishable goods are lumpy in the sense outlined by Randall and Stoll (1980). For example, the number of visits to a park or the expenditure on such visits can be changed freely, but there is only one Grand Canyon. ${ }^{1}$ Similar to Randall and Stoll (1980), we assume that there is a well-functioning market for perishable goods, so that we can study the associated price effects. On the other hand, the non-perishable goods are assumed to be public goods for which a market or a price may not exist. We instead focus on the quantity effects, the WTP/WTA and their relationship to $\mathrm{CV} / \mathrm{EV}$. We show that information and future learning

\footnotetext{
${ }^{1}$ There are exceptions: books and sport-cards are non-perishable but divisible goods.
} 
will have different consumer behavioral implications for the two sets of goods. However, in both cases we find that even in the presence of stable preferences, demand and WTP will shift through time as consumers trade off the advantage of delaying action (thereby collecting more information on the substitutes available and the value of the good) with the costs of delayed consumption.

This paper proceeds as follows. In the first section we extend the results of Zhao and Kling (2000, 2001) for the non-perishable goods case to a more general utility function to develop a general form for WTP and WTA in the dynamic setting. Importantly, we show that WTP and WTA can be different from $\mathrm{CV}$ and $\mathrm{EV}$, with the difference termed commitment costs. We conclude the nonperishable goods model case with a discussion of the implications of this finding for environmental valuation. Next, we consider the perishable goods model where we derive "information conditioned" Marshallian and Hicksian demand curves and show how they relate to each other and how they evolve as the consumers' information about the transaction changes. Here, commitment costs do not arise, as : a decision made in the first period has no carryover into the second period. Nonetheless, uncertainty and future learning may still drive a wedge between the observed consumer surplus (CS) and the true EV/CV, so that CS may not be bounded by EV and CV.

\section{A Model of Non-perishable Goods}

Consider an agent's decision about how much to pay to obtain more of a non-perishable good, or to accept to forgo the opportunity of increasing her consumption of this good. Let $U(x, y, \theta)$ be her utility function, where $x$ is the quantity of the non-perishable good, $y$ is the quantity of a composite good, the price of which is normalized to one, and $\theta$ is a parameter which affects the marginal utility of $x$. In our example, $\theta$ may reflect the air quality or accessibility at the Grand Canyon. Currently the agent does not know the value of $\theta$, only knowing that it is distributed 
according to $F(\cdot)$ on $\Theta \equiv\left[\theta_{l}, \theta_{h}\right]$. However, she will learn the true value of $\theta$ at the beginning of the next period. For instance, there may be a current study on the air quality at the Grand Canyon which will be released at the start of the next year.

We assume that $x$ and $y$ are normal goods, $U(\cdot)$ is continuous and differentiable in all its arguments, and $U_{x \theta} \neq 0$. Let $m$ be the agent's (constant) per period income. The non-perishable good $x$ is a public good, for which a market does not exist. Currently, the good exists at the level $x_{0}$. Thus the agent spends all her income on $y$ and obtains the utility $U\left(x_{0}, m, \theta\right)$. Note that because $y$ is a single composite good priced at 1 , the utility function $U(\cdot)$ is the same as the indirect utility function. For simplicity, we assume away consumption smoothing across periods. That is, the per period income $m$ cannot be shifted across periods. We will discuss the implications of this assumption later on.

\section{Willingness to Pay}

Suppose that a proposal is made to increase the amount of the non-perishable good to $x_{1}>x_{0}$, and the agent is asked to report her willingness to pay (WTP) for $x_{1}$. If the agent cannot learn about $\theta$ or ignores the learning opportunities, her (annualized) WTP is determined by

$$
E_{\theta} U\left(x_{0}, m, \theta\right) / r=E_{\theta} U\left(x_{1}, m-W T P^{n}, \theta\right) / r
$$

where $r$ is her discount rate and $W T P^{n}$ denotes the WTP under no learning. Implicitly, we are assuming that her WTP decision is irreversible: if she is committed to paying a certain amount, she will have to do so in every period in the future. Equation (1) indicates that $W T P^{n}$ is the same as the standard compensating variation $(\mathrm{CV})$, defined as

$$
E_{\theta} U\left(x_{0}, m, \theta\right)=E_{\theta} U\left(x_{1}, m-C V, \theta\right)
$$

However, if the agent recognizes her learning opportunities in the second period, her WTP will differ from $\mathrm{CV}=W T P^{n}$. To see this, we first determine her maximum WTP for $x_{1}$ when she knows 
she can delay her purchase until the next period. Let $c$ be a per period cost of $x_{1}$. We seek to determine the maximum $c$ she will agree today to pay in all periods. If she decides to pay in the current period, her expected payoff is

$$
V_{0}=E_{\theta} U\left(x_{1}, m-c, \theta\right) / r .
$$

If she waits until the second period when she knows the true value of $\theta$, she will pay $c$ if and only if $U\left(x_{1}, m-c, \theta\right) \geq U\left(x_{0}, m, \theta\right)$. In this case, she can avoid "purchasing" the good if it turns out not to be very valuable. Let $\Theta_{p}(c) \subseteq \Theta$ such that $U\left(x_{1}, m-c, \theta\right) \geq U\left(x_{0}, m, \theta\right)$ if and only if $\theta \in \Theta_{p}$, and let $\Theta_{n p}(c)=\Theta \backslash \Theta_{p}(c)$. Then her expected payoff of waiting to decide until the second period is

$$
\begin{aligned}
V_{1} & =E_{\theta} U\left(x_{0}, m, \theta\right)+\frac{1}{(1+r) r}\left[E_{\theta \in \Theta_{p}} U\left(x_{1}, m-c, \theta\right)+E_{\theta \in \Theta_{n p}} U\left(x_{0}, m, \theta\right)\right] \\
& =E_{\theta} U\left(x_{0}, m, \theta\right) / r+\frac{1}{(1+r) r} E_{\theta \in \Theta_{p}}\left[U\left(x_{1}, m-c, \theta\right)-U\left(x_{0}, m, \theta\right)\right]
\end{aligned}
$$

where $E_{\theta \in \Theta_{p}}$ represents expectation over the set $\Theta_{p}$ (which is not the conditional expectation).

It is clear from (3) and (4) that both $V_{0}$ and $V_{1}$ are monotone decreasing in $c$, and $V_{0}$ decreases in $c$ faster than $V_{1}$. Intuitively, as the cost of $x_{1}$ increases, there will be more values of $\theta$ such that the agent will decide not to purchase $x_{1}$. Her loss from the higher $c$ is thus lower than it would be without this opportunity to delay. In addition, $V_{0}>V_{1}$ at $c=0$ and $V_{0}<V_{1}$ at $c=\infty$ : due to discounting, the agent prefers to enjoy $x_{1}$ earlier if it costs nothing. If it is extremely costly, she is better off not buying $x_{1}$. Then, a unique $c$ exists that equates $V_{0}$ and $V_{1}$, and this unique value is the maximum value of $c$ such that $V_{0} \geq V_{1}$. Thus, the value is the agent's WTP today when the opportunity to delay and learn is present, denoted as $W T P^{l}$ where superscript $l$ represents learning:

$$
E_{\theta} U\left(x_{0}, m, \theta\right)=E_{\theta} U\left(x_{1}, m-W T P^{l}, \theta\right)-\beta E_{\theta \in \Theta_{p}}\left[U\left(x_{1}, m-W T P^{l}, \theta\right)-U\left(x_{0}, m, \theta\right)\right]
$$

where $\beta=1 / 1+r$ is the discount factor. 
Equation (5) is closely related to the quasi-option value literature (Arrow and Fisher 1974) and Dixit and Pindyck 1994. It can be rewritten as

$$
E_{\theta} U\left(x_{0}, m, \theta\right) / r+O V_{p}=E_{\theta} U\left(x_{1}, m-W T P^{l}, \theta\right) / r
$$

where $O V_{p}=(\beta / r) E_{\theta \in \Theta_{p}}\left[U\left(x_{1}, m-W T P^{l}, \theta\right)-U\left(x_{0}, m, \theta\right)\right]$ is the (quasi) option value associated with waiting. If the agent decides not to buy $x_{1}$ in the current period, she can still buy in the next period. Thus, the expected payoff of not buying today is the direct payoff $E_{\theta} U\left(x_{0}, m, \theta\right) / r$ plus the option value of the future decision $O V_{p}$.

Now it is straightforward to compare $W T P^{l}$ and $C V=W T P^{n}$. From (2) and (5), we know $W T P^{l}<C V$. The inequality is strict because if $W T P^{l}=C V$, by $(2), \Theta_{p}\left(W T P^{l}\right)$ is nonempty. ${ }^{2}$ The last term in (5) is then strictly positive, so (2) and (5) would contradict each other. In order for the agent to "buy" $x_{1}$ in the current period, the "price" of $x_{1}$ has to be lower to compensate for the lost opportunity of obtaining more information.

Define the commitment cost associated with WTP as $C C_{p}=C V-W T P^{l}$. It measures the reduction in the agent's willingness to pay in order to compensate for the commitment of deciding now and giving up future information gathering. From (2) and (6), we have

$$
E_{\theta} U\left(x_{1}, m-C V, \theta\right)=E_{\theta} U\left(x_{1}, m-C V+C C_{p}, \theta\right)-r O V_{p}
$$

Applying Taylor expansion around $m$, we know

$$
C C_{p}=\frac{r O V_{p}}{E_{\theta} U_{m}\left(x_{1}, m, \theta\right)}
$$

when $C V$ and $W T P^{l}$ are small. Thus, in the neighborhood of $m$, the commitment cost equals the annualized option value in monetary units. The option value measures the value of being able to wait for more information. That is, for the agent to buy in the current period, the lump sum

\footnotetext{
${ }^{2}$ Otherwise, if $\Theta_{p}(C V)=\emptyset, U\left(x_{1}, m-C V, \theta\right)>U\left(x_{0}, m, \theta\right)$ for all $\theta \in \Theta$, which implies that $E_{\theta} U\left(x_{1}, m-C V, \theta\right)>$ $E_{\theta} U\left(x_{0}, m, \theta\right)$, violating $(2)$.
} 
compensation she demands is $O V_{p}$ (c f. [6]). Of course, this compensation can also come in the form of annual utils of $r O V_{p}$. Dividing the annual utils by the (expected) marginal utility of income, we obtain the corresponding annual monetary transfer $r O V_{p} / E U_{m}$. But this transfer is precisely the lower "price" the agent demands for giving up the learning opportunity, or the commitment cost.

Equation (7) indicates that the results of the quasi-option value literature can be applied to commitment costs. For example, as the uncertainty of $F(\cdot)$ increases, the agent learns more about $\theta$ by waiting. Then the option value of waiting $O V_{p}$ rises, and so does $C C_{p}$. As the discount factor $\beta$ increases, the cost of waiting in terms of delayed consumption of $x_{1}$ is lower. Again, $O V_{p}$ and thus $C C_{p}$ increases. This can be written as follows.

Proposition 1 The commitment cost $C C_{p}$ is increasing in the level of uncertainty of $F(\cdot)$ and the discount factor $\beta$.

\section{Willingness to Accept}

Similar to the case of WTP, in the case of no learning, the agent's (annualized) WTA, i.e., her required compensation for continuing to consume $x_{0}$ instead of consuming $x_{1}$, is determined by

$$
E_{\theta} U\left(x_{0}, m+W T A^{n}, \theta\right) / r=E_{\theta} U\left(x_{1}, m, \theta\right) / r .
$$

Again, $W T A^{n}$ is the same as the equivalent variation (EV), defined in

$$
E_{\theta} U\left(x_{0}, m+E V, \theta\right)=E_{\theta} U\left(x_{1}, m, \theta\right) .
$$

Consider now the case when the agent can learn about $\theta$. Again, we need to determine the compensation she will accept in lieu of $x_{1}$. Suppose she is presented with the compensation level $w$. Her consumption will not increase from $x_{0}$ to $x_{1}$ if she accepts $w$. If she accepts now, her expected payoff is

$$
\pi_{0}=E_{\theta} U\left(x_{0}, m+w, \theta\right) / r .
$$


If she waits until the second period when she observes the true value of $\theta$, she will choose to accept only when $U\left(x_{0}, m+w, \theta\right) \geq U\left(x_{1}, m, \theta\right)$. That is, she can accept the compensation only when $x_{1}$ turns out to have low value. Define $\Theta_{a}(w) \subseteq \Theta$ such that $U\left(x_{0}, m+w, \theta\right) \geq U\left(x_{1}, m, \theta\right)$ if and only if $\theta \in \Theta_{a}(w)$. Let $\Theta_{n a}(w)=\Theta \backslash \Theta_{a}(w)$. Then her expected payoff of waiting is

$$
\begin{aligned}
\pi_{1} & =E_{\theta} U\left(x_{1}, m, \theta\right)+\frac{1}{(1+r) r}\left[E_{\theta \in \Theta_{a}} U\left(x_{0}, m+w, \theta\right)+E_{\theta \in \Theta_{n a}} U\left(x_{1}, m, \theta\right)\right] \\
& =E_{\theta} U\left(x_{1}, m, \theta\right) / r+\frac{1}{(1+r) r} E_{\theta \in \Theta_{a}}\left[U\left(x_{0}, m+w, \theta\right)-U\left(x_{1}, m, \theta\right)\right] .
\end{aligned}
$$

Note that in the first period, while she is waiting for the new information, she enjoys $x_{1}$ but does not receive the compensation $w$.

Both $\pi_{0}$ and $\pi_{1}$ are increasing in $w$, but $\pi_{0}$ increases at a faster rate. Further, $\pi_{0}>\pi_{1}$ as $w \rightarrow \infty$ and $\pi_{0}<\pi_{1}$ as $w \rightarrow 0$. Thus there is a unique value of $w$ that equates $\pi_{0}$ and $\pi_{1}$. It is also the minimum value of $w$ so that $\pi_{0}>\pi_{1}$, or the minimum value of compensation needed for the agent to accept in the current period. This value is the agent's WTA with learning, given by

$$
E_{\theta} U\left(x_{1}, m, \theta\right)=E_{\theta} U\left(x_{0}, m+W T A^{l}, \theta\right)-\beta E_{\theta \in \Theta_{a}}\left[U\left(x_{0}, m+W T A^{l}, \theta\right)-U\left(x_{1}, m, \theta\right)\right] .
$$

Comparing (9) and (12), we find that $W T A^{l}>E V$ : the agent demands additional compensation in the form of higher WTA for deciding in the current period and forgoing the future learning opportunities. Again, (12) can be rewritten as

$$
E_{\theta} U\left(x_{1}, m, \theta\right) / r=E_{\theta} U\left(x_{0}, m+W T A^{l}, \theta\right) / r-O V_{a},
$$

where $O V_{a}=(\beta / r) E_{\theta \in \Theta_{a}}\left[U\left(x_{0}, m+W T A^{l}, \theta\right)-U\left(x_{1}, m, \theta\right)\right]$ is the (quasi) option value associated with waiting. Define the associated commitment cost as $C C_{a}: C C_{a}=W T A^{l}-E V$. From (9) and (13), we know

$$
C C_{a}=\frac{r O V_{a}}{E_{\theta} U_{m}\left(x_{0}, m, \theta\right)}
$$

Therefore, we have the following. 
Proposition 2 The commitment cost $C C_{a}$ is increasing in the level of uncertainty of $F(\cdot)$ and the discount factor $\beta$.

\section{The Effects of Functional Forms and Consumption Smoothing}

The size of the commitment costs depends on the curvature of the utility function $U(\cdot)$, in addition to uncertainty and $\beta$ identified in Propositions 1 and 2. However, unlike Hanemann (1991), the substitutability between $x$ and $y$ may not significantly affect the size of the commitment costs or the divergence between the observed $W T P^{l}$ and $W T A^{l}$. From (7) and (14), for the same option values, the commitment costs are smaller the higher the expected marginal utility of income. The option values are conditional values of information (Hanemann 1989), and they can be strictly positive even when there is perfect substitutability between $x$ and $y$.

To illustrate the different effects of the substitutability on $\mathrm{CV} / \mathrm{EV}$ and $W T P^{l} / W T A^{l}$, we study numerically the modified CES utility function $U(x, y, \theta)=\theta \frac{x^{\rho}}{\rho}+\frac{y^{\rho}}{\rho}$ where $0<\rho<1$ is the elasticity of substitution. Figure 1 shows the effects of $\rho$ for $m=10, x_{0}=1, x_{1}=2$, and $\theta \in$ Uniform[0,4]. In Panel (a), the four curves are, from the top, $W T A^{l}, E V, C V$ and $W T P^{l}$ values. Thus, as predicted by Hanemann (1991), the divergence between $E V$ and $C V$ decreases as the elasticity of substitution rises. Further, the $W T P^{l} / W T A^{l}$ divergence also goes down, possibly due to the fact

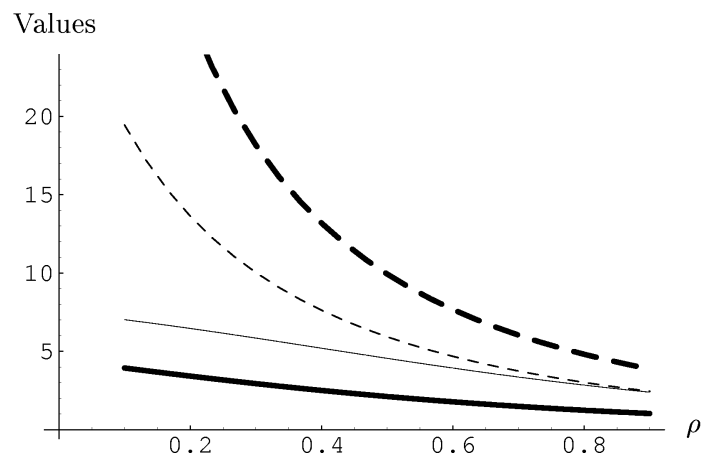

(a) Values of Welfare Measures

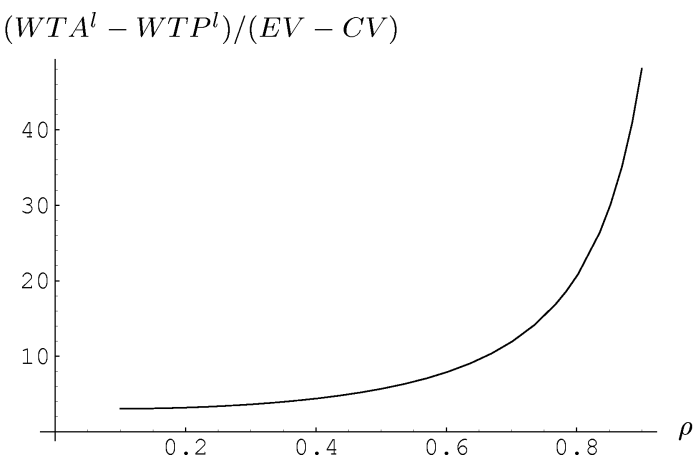

(b) WTP/WTA Relative to CV/EV Divergences

Figure 1: The Effects of Substitutability on the Divergence 
that the marginal utility of income increases in $\rho$. However, the ratio between the $W T P^{l} / W T A^{l}$ and $C V / E V$ divergences increases in $\rho$. That is, as the elasticity of substitution rises, the observed WTP/WTA divergence exaggerates the true CV/EV divergence proportionally more.

Now we discuss the implications of our assumption that there is no intertemporal consumption smoothing. Since the WTP and WTA are expressed as the per period payments, the only possible consumption smoothing occurs in the first period if the agent decides to wait for more information. For example, in the case of WTP, if the agent decides to buy $x_{1}$ in the first period, her consumption of $y$ is $m-w t p$ in every period. Given the stationary utility, no consumption smoothing is needed. However, if she decides to wait until period two to buy, her consumption of $y$ in period one will be more than in later periods. She may want to save some income in period one for consumption later. As a result, the expected payoff of waiting, and thus the option value of waiting, will be higher if consumption smoothing is allowed. From the relationship between $C C_{p}$ and the option value, we know $C C_{p}$ will be higher. Thus, the assumption of no consumption smoothing reduces the divergence between $W T P^{l}$ and $W T A^{l}$, while not affecting that between $W T P^{n}$ and $W T A^{n}$.

\section{Implications for Welfare Measurement}

The results presented above are quite intuitive. They indicate that the opportunity to delay a purchase or sale until better information is available about the precise value of the good in question is valuable. To forgo this option, the consumer must be compensated (in the form of a lower price for a buyer and a higher price for a seller). When the agent's current choices have long-run effects that are hard to reverse, and when there are future learning opportunities that will make the choices more "intelligent" later, the agent's current $W T A^{n}$ and $W T A^{l}$ are typically different from the static $\mathrm{CV}$ or EV. Although the magnitude of this difference is an empirical question, several important implications for welfare measurement of environmental goods emerge. 
First, as discussed in Zhao and Kling (2001, 2000), the presence of commitment costs can explain the observed divergence between $W T P^{l}$ and $W T A^{l}$ in experimental and contingent valuation markets. Like the explanation provided by Hanemann (1991), our theory suggests that these divergences are consistent with stable preferences and optimizing behavior. Specifically, if a commitment cost is present in either or both of the $W T P^{l}$ or $W T A^{l}$ values, there will be an observed difference between them that exceeds the amount that income effects or Hanemann's substitution effects can generate. Since the explanation for how commitment costs can generate this divergence is discussed elsewhere (Zhao and Kling 2001), we focus here on the implications of this result for applied welfare measurement.

The widespread use of $W T P^{l}$ in contingent valuation experiments rather than $W T A^{l}$ appears mainly due to the "large" values of many $W T A^{l}$ estimates (NOAA report). If these high $W T A^{l}$ estimates are due to large commitment costs associated with "selling" the environment (as would be the case if survey respondents or experimental subjects feel that once they give up the environmental quality, it will be difficult or impossible to re-obtain it), then this observation provides justification for the use of $W T P^{l}$, rather than $W T A^{l}$, as a better approximation for CV/EV in stated preference studies. But, the use of $W T P^{l}$ as a closer approximation to $\mathrm{CV} / \mathrm{EV}$ implies that $\mathrm{CV} / \mathrm{EV}$ are the correct welfare measure for policy purposes instead of the $W T P^{l}$ or $W T A^{l}$ values that contain commitment costs.

When is expected $\mathrm{CV}$ or EV the correct welfare measure to use and when is $W T P^{l}$ or $W T A^{l}$ appropriate? In using a WTP question to elicit the value of a public good in a contingent valuation survey, the researcher may unwittingly induce respondents to include commitment costs in their responses. Contingent valuation surveys generally ask action questions (e.g., are you willing to pay $x$ dollars to keep this park, or how much would you be willing to pay to keep the park 
open?), instead of direct value questions (e.g., how much surplus would you receive if this park is kept open?). In the absence of commitment costs, these two questions (and their answers) are equivalent, but when values are uncertain and learning is possible, asking for WTP or WTA comingles commitment costs with the implicit value of the good. So, for example, in answering a WTA question about giving up a local park, respondents may include a large commitment cost because they have not investigated the characteristics of other local parks in the area that might provide good substitutes. Were they given adequate time to investigate these alternatives, the commitment cost might disappear. But a standard contingent valuation survey asks for a value now; under that circumstance the reported $W T A^{l}$ may contain a large commitment costs.

In contrast, some decisions are inherently characterized by uncertainty and irreversibility and contain commitment costs that are independent of the experimental or survey design. For example, the decision to build a dam contains a significant amount of uncertainty regarding the benefits and costs of the change. In such a case, a contingent valuation survey that accurately replicates the decision framework will elicit $W T A^{l}$ and $W T P^{l}$ measures that contain the commitment costs. But these costs should enter the welfare calculations; thus, $W T A^{l}$ or $W T P^{l}$ are in fact the appropriate welfare measures.

The main point is that whether researchers are using contingent valuation studies, laboratory experiments, or market transactions data, they need to be cognizant of the possible inclusion of commitment costs in $W T P^{l}$ and/or $W T A^{l}$. Fundamentally, if policy-relevant option values cause the divergence between $W T P^{l}$ and $W T A^{l}$, then the reliance on $W T P^{l}$ when $W T A^{l}$ is the more appropriate measure will generate inefficient resource allocations. On the other hand, if the divergence between $W T P^{l}$ and $W T A^{l}$ arises from analysis-induced commitment costs that do not have a basis in the true situation, the use of either $W T P^{l}$ or $W T A^{l}$ may yield inefficient outcomes. Again, the key point is that analysts must understand that option values may arise in $W T P^{l}$ and/or $\mathrm{WTA}^{1}$ values and they must consciously choose which measure is appropriate. 


\section{A Model of Perishable Goods}

For simplicity we consider a two-period model. There is a market for $x$, the price of which is $p$. The choice of $x$ in the current period does not directly restrict its consumption level in the second period.

Further, we assume that (i) $U(\cdot)$ is linear in $\theta$, (ii) $U_{x \theta} \geq 0$, and (iii) $\frac{d}{d \theta}\left(\frac{U_{x}}{U_{y}}\right) \neq 0$. Assumption (i) is made for simplicity: uncertainty in $\theta$ does not change the utility directly if the consumption bundle is unaffected. This assumption is not as restrictive as it first appears since reparameterization of the random parameter is possible. For example, if $U(\cdot)=f(\tilde{\theta}) u(x, y)$ where $f(\cdot)$ is not linear, we can redefine $\theta=f(\tilde{\theta})$ to achieve linearity in $\theta$. Assumptions (ii) and (iii) guarantee that $\theta$ does matter in the agent's choice of the consumption bundles: $\theta$ affects the marginal utility of $x$ and the marginal rate of substitution between $x$ and $y$. Thus, given the same income level, the agent will choose a different bundle if $\theta$ changes. Without these conditions, a model of learning is uninteresting. Note that (iii) does exclude some common utility functions, such as the Cobb-Douglas utility function $U(x, y, \theta)=\theta x^{\alpha} y^{\beta}$. Learning about $\theta$ is irrelevant for such utility functions since it does not affect the agent's choices.

\section{The Case of No Learning}

Consider first the case where the agent cannot learn or ignores the learning possibilities in deciding her consumption bundle in the first period. Her decision problem is

$$
\begin{aligned}
& \max _{x_{1}, y_{1}, x_{2}, y_{2}} U\left(x_{1}, y_{1}, \bar{\theta}\right)+\beta U\left(x_{2}, y_{2}, \bar{\theta}\right) \\
& \text { s.t. } \quad p x_{1}+y_{1}+\beta\left(p x_{2}+y_{2}\right)=M,
\end{aligned}
$$

where $\bar{\theta}=E \theta$ is the expected value of $\theta$, and $M$ is the agent's lifetime income. Note that $E_{\theta} U(x, y, \theta)=U(x, y, \bar{\theta})$ because $U(\cdot)$ is assumed to be linear in $\theta$. 
From the first-order conditions of (15), we know

$$
\frac{U_{x_{1}}}{U_{y_{1}}}=\frac{U_{x_{2}}}{U_{y_{2}}}=p, \quad U_{x_{1}}=U_{x_{2}}, \quad \text { and } \quad U_{y_{1}}=U_{y_{2}}
$$

Thus, $x_{1}=x_{2}=x$ and $y_{1}=y_{2}=y$ : the agent chooses the same consumption bundle in both periods. Substituting the solution back into the budget constraint in (15), we know the agent allocates her income between the two periods equally, adjusted by the discounting factor. In particular, the choice of her first period's consumption bundle in (15) can be represented as

$$
\begin{gathered}
\max _{x_{1}, y_{1}} U\left(x_{1}, y_{1}, \bar{\theta}\right) \\
\text { s.t. } \quad p x_{1}+y_{1}=M_{1} \equiv \frac{M}{1+\beta} .
\end{gathered}
$$

Note that the income allocated to the first period $M_{1}$ is independent of the price $p$ or the value of $\theta$. This is where the simplifying assumption of linearity in $\theta$ is particularly useful. In studying the agent's demand function for the goods in the first period, we can ignore the dynamic nature of the problem, and simply work in the static framework of (16). As a result, the standard results on Marshallian and Hicksian demand functions, such as the associated expenditure and indirect utility functions and the Slutsky equation, apply to the dynamic model without learning through (16).

To be comparable with the case of learning about $\theta$, the dynamic model in (15) can also be written recursively in the following form:

$$
\max _{x_{1}, y_{1}}\left\{U\left(x_{1}, y_{1}, \bar{\theta}\right)+\beta \max _{x_{2}, y_{2}} E_{\theta}\left\{U\left(x_{2}, y_{2}, \theta\right), \text { s.t. } \beta\left(p x_{2}+y_{2}\right)=M-p x_{1}-y_{1}\right\}\right\}
$$

Given $\left\{x_{1}, y_{1}\right\}$, the agent maximizes her expected utility subject in the second period to the income of $M-p x_{1}-y_{1}$. Thus, what affects the agent's second period choices is the expenditure of the first period, instead of the particular bundles chosen. 


\section{The Case of Learning}

Consider now the case where the agent learns about the true value of $\theta$ at the beginning of the second period. Her decision problem then becomes

$$
\max _{x_{1}, y_{1}}\left\{U\left(x_{1}, y_{1}, \bar{\theta}\right)+\beta E_{\theta} \max _{x_{2}, y_{2}}\left\{U\left(x_{2}, y_{2}, \theta\right), \text { s.t. } \beta\left(p x_{2}+y_{2}\right)=M-p x_{1}-y_{1}\right\}\right\},
$$

where the difference between (18) and (17) is the location of the expectation operator in the second term: the maximization in the former is conducted after observing $\theta$. Let $V\left(p, M-p x_{1}-y_{1}, \theta\right)$ be the indirect utility function in the second period, and let $M_{2}=\left(M-p x_{1}-y_{1}\right) / \beta$. The two objective functions can be represented as

$$
\begin{gathered}
\max _{x_{1}, y_{1}} U\left(x_{1}, y_{1}, \bar{\theta}\right)+\beta E_{\theta} V\left(p, M_{2}, \theta\right) \\
\max _{x_{1}, y_{1}} U\left(x_{1}, y_{1}, \bar{\theta}\right)+\beta V\left(p, M_{2}, \bar{\theta}\right) .
\end{gathered}
$$

The expected payoff is higher under learning as $V(\cdot)$ is convex in $\theta$, i.e., $E_{\theta} V\left(p, M_{2}, \theta\right)>V\left(p, M_{2}, \bar{\theta}\right) .^{3}$

This simply means that when the consumer has the opportunity to make a decision under better information, she can achieve higher utility.

The demand functions for $x_{1}$ and $y_{1}$ under learning and no learning are given by the respective first-order conditions:

$$
\begin{array}{rrr}
\text { (Learning) } & U_{x}\left(x_{1}, y_{1}, \bar{\theta}\right)=p E_{\theta} V_{m}\left(p, M_{2}, \theta\right) ; & U_{y}\left(x_{1}, y_{1}, \bar{\theta}\right)=E_{\theta} V_{m}\left(p, M_{2}, \theta\right) \\
\text { (No learning) } & U_{x}\left(x_{1}, y_{1}, \bar{\theta}\right)=p V_{m}\left(p, M_{2}, \bar{\theta}\right) ; & U_{y}\left(x_{1}, y_{1}, \bar{\theta}\right)=V_{m}\left(p, M_{2}, \bar{\theta}\right) .
\end{array}
$$

Similar to the case of no learning, the allocation of the first period's expenditure between $x_{1}$ and $y_{1}$ is independent of the second period's consumption or learning: $U_{x} / U_{u}=p$. Thus, learning affects the optimal $x_{1}$ or $y_{1}$, only through changing the portion of the total income $M$ that is allocated to the first period.

\footnotetext{
${ }^{3}$ To show the convexity of $V(\cdot)$, let $\left\{x^{1}, y^{1}\right\}$ be the optimal second period's bundle given $\theta^{1}$. Consider another value of $\theta, \theta^{2}>\theta^{1}$. If the consumption bundle is fixed at $\left(x^{1}, y^{1}\right)$, the payoff would change (say, increase, without loss of generality) linearly in $\theta$. Thus, $V$ will increase more than linearly in $\theta$ as the optimal consumption bundle $\left(x^{2}, y^{2}\right)$ will generate higher payoff than $\left(x^{1}, y^{1}\right)$ at $\theta^{2}$. That is, $V(\cdot)$ is convex in $\theta$.
} 
To show how income $M$ is allocated between the two periods and how the allocation depends on learning, note that the no-learning optimization problem (15) can be rewritten as

$$
\begin{aligned}
& \max _{M_{1}, M_{2}} V\left(p, M_{1}, \bar{\theta}\right)+\beta V\left(p, M_{2}, \bar{\theta}\right) \\
& \text { s.t. } \quad M_{1}+\beta M_{2}=M .
\end{aligned}
$$

and the learning problem (18) can be rewritten as

$$
\begin{aligned}
& \max _{M_{1}, M_{2}} V\left(p, M_{1}, \bar{\theta}\right)+\beta E_{\theta} V\left(p, M_{2}, \theta\right) \\
& \text { s.t. } \quad M_{1}+\beta M_{2}=M .
\end{aligned}
$$

It is clear from (20) that the optimal income allocation without learning is $M_{1}=M_{2}$. However, the allocation with learning must satisfy the following first-order condition:

$$
V_{m}\left(p, M_{1}, \bar{\theta}\right)=E_{\theta} V_{m}\left(p, M_{2}, \theta\right)
$$

Because $\mathrm{V}(\cdot)$ is increasing and concave in $M, M_{2}>M_{1}$ if $V_{m}(\cdot)$ is convex in $\theta$ and $M_{2}<M_{1}$ if $V_{m}(\cdot)$ is concave in $\theta$.

Proposition 3 If the indirect utility function is such that $V_{m}(p, m, \theta)$ is convex (or concave) in $\theta$, learning about $\theta$ reduces (or raises) the income allocated to the first period, thereby shifting in (or out) the (Marshallian) demand functions of both $x_{1}$ and $y_{1}$. If $V_{m}(\cdot)$ is linear in or independent of $\theta$, learning does not affect the income allocation.

Intuitively, because income is "more useful" when the agent has more information about $\theta$, and because the extra information occurs in the second period, we might expect that income will be moved from the first period into the second to take advantage of this efficiency, i.e., $M_{2}>M_{1}$. Suppose the agent is given a little more income. If she simply ignores the new information about $\theta$, she will allocate the additional income according to the ratio $U_{x}(x, y, \bar{\theta}) / U_{y}(x, y, \bar{\theta})=p$, and will obtain more utility from additional consumption of $x$ and $y$. But if she recognizes the information about $\theta$, she 
will allocate the additional income differently according to the realized value of $\theta$. In expectation, she should obtain more utility from the additional income if she puts it into the second period because it is allocated more "efficiently." However, this result is not assured. It is also possible that it could be efficient to move income from the second period into the first as the increase in efficiency in the second period is equivalent to an increase in total purchasing power. Since the efficiency enhancement in the second period allows higher purchasing power in that period, she may prefer to allocate more nominal income to the first period to raise that purchasing power.

Thus, specific functional forms and parameter values may be needed to characterize the convexity of $V_{m}(\cdot)$ in $\theta$. Below we present two examples where $V_{m}(\cdot)$ is linear or convex in $\theta$. We have not yet been able to identify functional forms where $V_{m}(\cdot)$ is concave in $\theta$.

Example 1: Consider a quasi-linear utility function $U(x, y, \theta)=\theta \ln x+y$. In this case, it is straightforward to verify that the indirect utility function is $V(p, m, \theta)=\theta \ln \frac{\theta}{p}+M-\theta$. Although $V(\cdot)$ is convex in $\theta, V_{m}=1$ is independent of $\theta$. Thus, learning does not affect the demand functions of $x_{1}$ or $y_{1}$.

Example 2: Consider the modified CES utility function $U(x, y, \theta)=\theta \frac{x^{\rho}}{\rho}+\frac{y^{\rho}}{\rho}$ where $0<\rho<1$ is the elasticity of substitution. We can show that the indirect utility function is $V(p, m, \theta)=$ $A(p, \theta) m^{\rho}$ where

$$
A(p, \theta)=\frac{\theta}{\rho}\left(\frac{1}{p+\theta^{-1 / 1-\rho} p^{1 / 1-\rho}}\right)^{\rho}+\frac{1}{\rho}\left(\frac{1}{1+\theta^{1 / 1-\rho} p^{-\rho / 1-\rho}}\right)^{\rho} .
$$

$V(\cdot)$ being convex in $\theta$ implies that $A(p, \theta)$ is convex in $\theta$. Thus $V_{m}=A \rho m^{\rho-1}$ is also convex in $\theta$. In this case, learning about future $\theta$ reduces the income allocated to period one and thus the demand functions of $x_{1}$ and $y_{1}$.

Let $x_{1}^{l}\left(p, M_{1}\right)$ be the (Marshallian) demand function of $x_{1}$ under learning, and $x_{1}^{n}\left(p, M_{1}\right)$ be that under no learning. Let $\Delta(p, M)$ be the "additional" net income allocated away from period 
one to period two, determined in (22). Then we know that

$$
x_{1}^{l}\left(p, M_{1}\right)=x_{1}^{n}\left(p, M_{1}-\Delta(p, M)\right) .
$$

Suppose $V_{m}(\cdot)$ is indeed convex in $\theta$. Then as the agent expects more information in the second period, e.g., as the variance of $F(\cdot)$ increases, less income will be allocated to the first period and the demand functions of $x_{1}$ and $y_{1}$ will be shifted more inward. In a multiple-period model, as the agent's information increases over time, her demand function also shifts out. Further, providing more information to the agent will increase the demand in the early periods.

These results establish the fact that when a consumer is forward looking, her demand function and associated welfare measures will shift through time as she acquires more information. This occurs despite stable preferences, prices, and income.

\section{The Effects of a Price Change}

In neoclassical economic theory, the demand and welfare responses to price changes based on $x_{1}^{n}(p, m)$ have been analyzed through the Slutsky equation, willingness to pay and accept, and compensating and equivalent variation. In this section, we study how these standard results based on $x_{1}^{n}(p, m)$ need to be modified for $x_{1}^{l}(p, m)$ given learning.

When $p$ changes, in addition to the standard income and substitution effects, the income allocation across periods may also change. From (22), we know $V_{m}\left(p, M_{1}-\Delta, \bar{\theta}\right)=E_{\theta} V_{m}\left(p, M_{2}+\Delta / \beta, \theta\right)$. The implicit function theorem implies that

$$
\frac{\partial \Delta(p, m)}{\partial p}=\frac{V_{m p}(\cdot, \cdot, \bar{\theta})-E_{\theta} V_{m p}(\cdot, \cdot, \theta)}{V_{m m}(\cdot, \cdot, \bar{\theta})+E_{\theta} V_{m m}(\cdot, \cdot, \theta) / \beta}
$$

Thus, similar to Proposition 3, the allocation depends on whether $V_{m p}(p, m, \theta)$ is convex or concave in $\theta$. Higher $p$ reduces (or increases) $M_{1}$ if $V_{m p}(\cdot)$ is convex (or concave) in $\theta$. That is, the allocation depends on whether learning makes the additional income "more useful" as $p$ increases. 


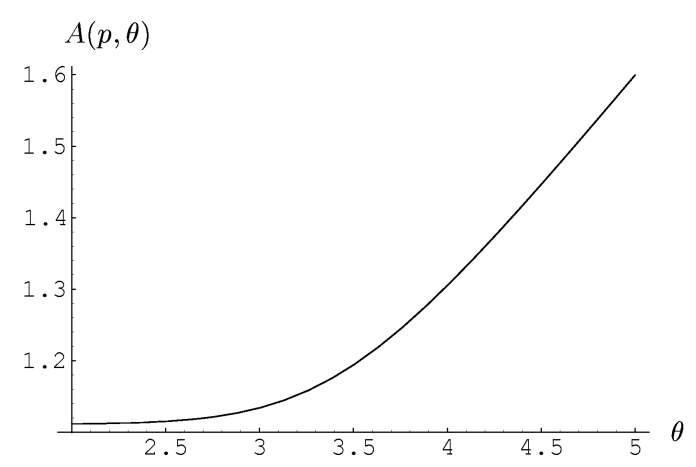

(a) $V_{m}(\cdot)$

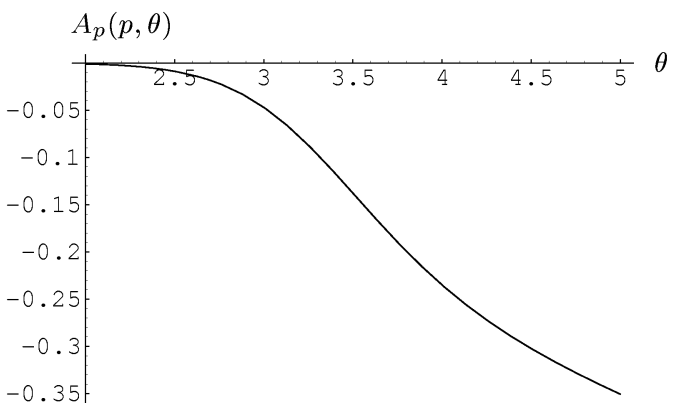

(b) $V_{m p}(\cdot)$

Figure 2: The Convexity and Concavity of $V_{m}(\cdot)$ and $V_{m p}(\cdot)$ in $\theta$

The convexity of $V_{m p}$ depends on the specific utility function and parameter values. Intuitively, as $p \rightarrow \infty$, little will be spent on $x$ and a further increase in $p$ will not matter much, regardless of the value of $\theta$. More information about $\theta$ will not affect the allocation of the income between $x$ and $y$, or the response of the allocation to the price changes. Thus, learning is of little value and $V_{m p}(\cdot)$ should be close to being linear in $\theta$. Similarly, as $p \rightarrow 0$, most of the income will be spent on $x$, regardless of small increases in $p$ or the value of $\theta$. Again, learning does not matter much in the allocation of income between $x$ and $y$ and in the response of the allocation to $p . V_{m p}(\cdot)$ should again be close to being linear in $\theta$.

In our two examples, $V_{m p}(\cdot)$ is independent of $\theta$ if $U(\cdot)$ is quasi-linear, and it may be convex or concave depending on the value of $p$. Figure 2 illustrates the latter example with parameter values $\rho=0.9$ and $p=4$. It is clear that while $A(\cdot)$, i.e., $V_{m}(\cdot)$, is always convex in $\theta, A_{p}(\cdot)$, or $V_{m p}(\cdot)$, can be convex and concave in $\theta$.

If $V_{m p}(\cdot)$ is not linear or independent of $\theta$, the Slutsky equation needs to be extended to incorporate the additional effect of a price change on the income allocation between the two periods. Let $h_{1}(p, u)$ be the Hicksian demand function for $x_{1}$ without learning. The standard Slutsky equation 


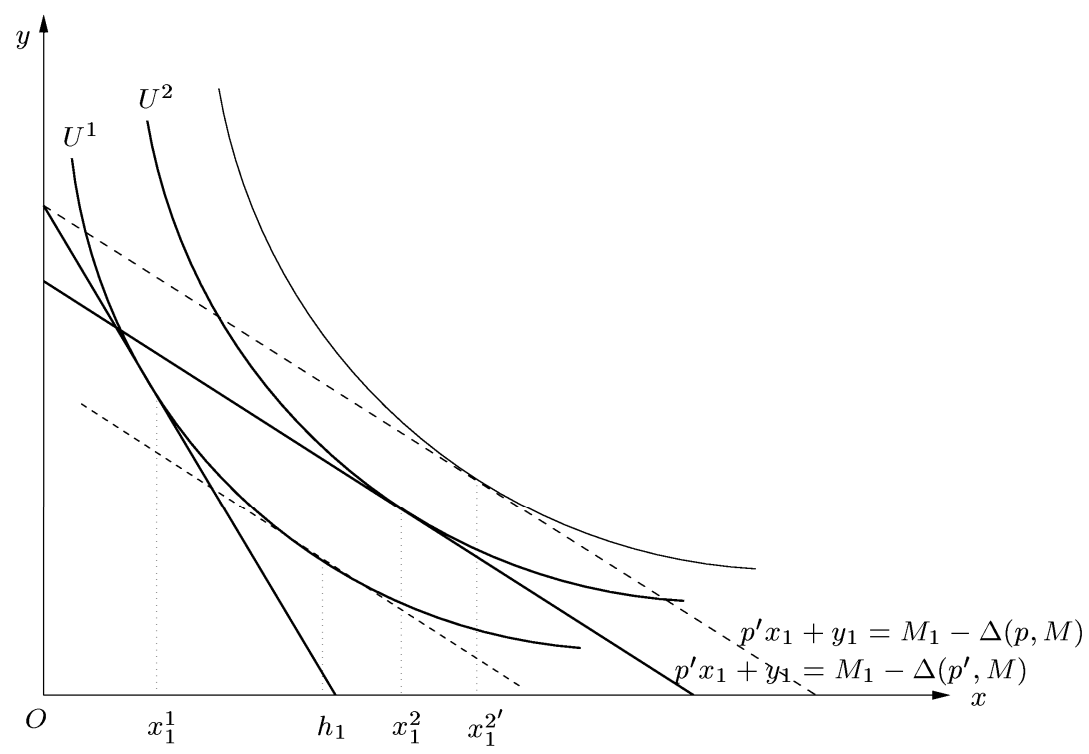

Figure 3: The Effects of a Decrease in $p$

relating $x_{1}^{n}$ and $h_{1}$ is

$$
\frac{\partial x_{1}^{n}\left(p, M_{1}\right)}{\partial p}=\frac{\partial h_{1}(p, u)}{\partial p}-\frac{\partial x_{1}^{n}\left(p, M_{1}\right)}{\partial m} x_{1}^{n} .
$$

From (23), we know

$$
\begin{aligned}
\frac{\partial x_{1}^{l}\left(p, M_{1}\right)}{\partial p} & =\frac{\partial x_{1}^{n}\left(p, M_{1}-\Delta\right)}{\partial p}-\frac{\partial x_{1}^{n}\left(p, M_{1}-\Delta\right)}{\partial m} \frac{\partial \Delta(p, M)}{\partial p} \\
& =\frac{\partial x_{1}^{n}\left(p, M_{1}-\Delta\right)}{\partial p}-\frac{\partial x_{1}^{l}\left(p, M_{1}\right)}{\partial m} \frac{\partial \Delta(p, M)}{\partial p},
\end{aligned}
$$

where the second equality follows from (23). Substituting the previous equation into this one, and noting that $x_{1}^{n}\left(p, M_{1}-\Delta\right)=x_{1}^{l}\left(p, M_{1}\right)$, we obtain the modified Slutsky equation for $x_{1}^{l}$ :

$$
\frac{\partial x_{1}^{l}\left(p, M_{1}\right)}{\partial p}=\frac{\partial h_{1}(p, u)}{\partial p}-\frac{\partial x_{1}^{l}\left(p, M_{1}\right)}{\partial m}\left(x_{1}^{l}+\frac{\partial \Delta(p, M)}{\partial p}\right),
$$

where $u$, the utility level, is fixed at $V\left(p, M_{1}-\Delta, \bar{\theta}\right)$. Thus, in addition to the standard substitution and income effects associated with a price increase, there is an income reallocation effect. If $V_{m p}(\cdot)$ is concave in $\theta$, lower $p$ would reduce $M_{1}$, offsetting the increase in $x_{1}$ due to the standard substitution and income effects.

Figure 3 illustrates these effects graphically for the case when $V_{m p}(\cdot)$ is concave in $\theta$. As $p$ 
Environmental Valuation U nder D ynamic C onsumer B ehavior / 21

decreases to $p^{\prime}$, the income allocated to the first period decreases. The increase in the consumption of $x_{1}$ is lower than without the income reallocation effect. The distance between $x_{1}^{1}$ and $h_{1}$ measures the substitution effect, while that between $h_{1}$ and $x_{1}^{2^{\prime}}$ measures the traditional static income effect. However, as $p$ changes to $p^{\prime}$, income allocated to the first period decreases, shifting the budget constraint in and moving the consumption of $x$ to $x_{1}^{2}$. This distance is due to the learning effect.

\section{Welfare Measurement}

Consider two decision environments an agent faces. In the first case, she is allowed to gather complete information about $\theta$ in the beginning of the first period. Thus, her decision problem is without uncertainty and is the same as the problem in (15) with $\bar{\theta}$ replaced by the true $\theta$. In the second case, she is forced to make her first-period decision without complete information but gathers the information only in the second period. Her decision problem is the same as in (18).

When answering a contingent valuation or contingent behavior question, the latter case could be viewed as applicable. In this situation, she is asked to respond to a set of questions in a limited timeframe, forgoing the opportunities of gathering more information about the environment amenity being valued or its substitutes and complements. Suppose the agent is asked to consider the introduction of a new park near her home and she is asked how many trips she would take to that park next year if the park were to open . Equivalently, she might be asked how much she would be willing to pay next year to visit the park. As is typical of contingent behavior or valuation questions, she would be expected to provide this answer in a short time period, either immediately if the survey is done via phone or in a few days if it is a mail survey. In any event, she is likely to provide a response to the question before she has gathered as much information about the prospective park as she would if she actually were to make the decision about how many trips to take to the park. 
Suppose that she responds to the survey, recognizing that there is potential for learning in the second period. She provides an answer based on $x_{1}^{l}(p, m)$ as illustrated in Figure 3 (the figure is drawn assuming that $V_{m}(\cdot)$ is convex in $\theta$ ). Suppose that everyone in the sample responds to this hypothetical question in the same way. Then the demand function estimated from the survey data will reflect the dynamic nature of the agents' decisions, and the analyst will have data to estimate $x_{1}^{l}(p, m)$. However, the "true" demand function, in expectation, should be $x_{1}^{n}(p, m)$. From (23), we know that $x_{1}^{l}(p, m)<x_{1}^{n}(p, m)$, as illustrated in Figure 4. Survey restrictions in this case result in underestimation of the demand and value of CV/EV for the environmental good. In this scenario, the fact that a response is elicited from the respondents before they have time to complete their information set generates an underestimate of the value and the observed use.

However, in some cases, the lack of learning opportunity is not imposed by the survey instrument but rather is inherent in the nature of the problem. For example, the agent may be actively thinking about visiting the park, and she will not have time to gather the relevant information before making her decision. In this case, the estimated demand $x_{1}^{l}\left(p, M_{1}\right)$ is in fact the relevant function for welfare measurement. The Hicksian demand functions associated with the two price levels $p_{0}$ and $p_{1}$ are $h_{1}\left(p, u^{0}\right)$ and $h_{1}\left(p, u^{1}\right)$, where $u^{0}=V\left(p_{0}, M_{1}-\Delta\left(p_{0}, M\right), \bar{\theta}\right)$ and $u^{1}=V\left(p_{1}, M_{1}-\Delta\left(p_{1}, M\right), \bar{\theta}\right.$.

Not surprisingly, in the case where survey restrictions result in the inappropriate use of $x_{1}^{l}\left(p, M_{1}\right)$, the true compensating and equivalent variation measures may not bound the estimated consumer surplus. It is possible that the estimated CS is lower than both CV and EV for the associated price change. That is, the standard Willig bounds may not work once learning is introduced. In our example, the observed CS is $p_{0} a g p_{1}$, while $\mathrm{CV}$ is $p_{0} c f p_{1}$ and $\mathrm{EV}$ is $p_{0} d e p_{1}$.

The implications for welfare measurement described here are similar to the non-perishable goods model in that the requirement imposed in surveys (and experiments) to form a willingness to pay or accept value without adequate time to learn can, in both cases, lead to biased estimates of 


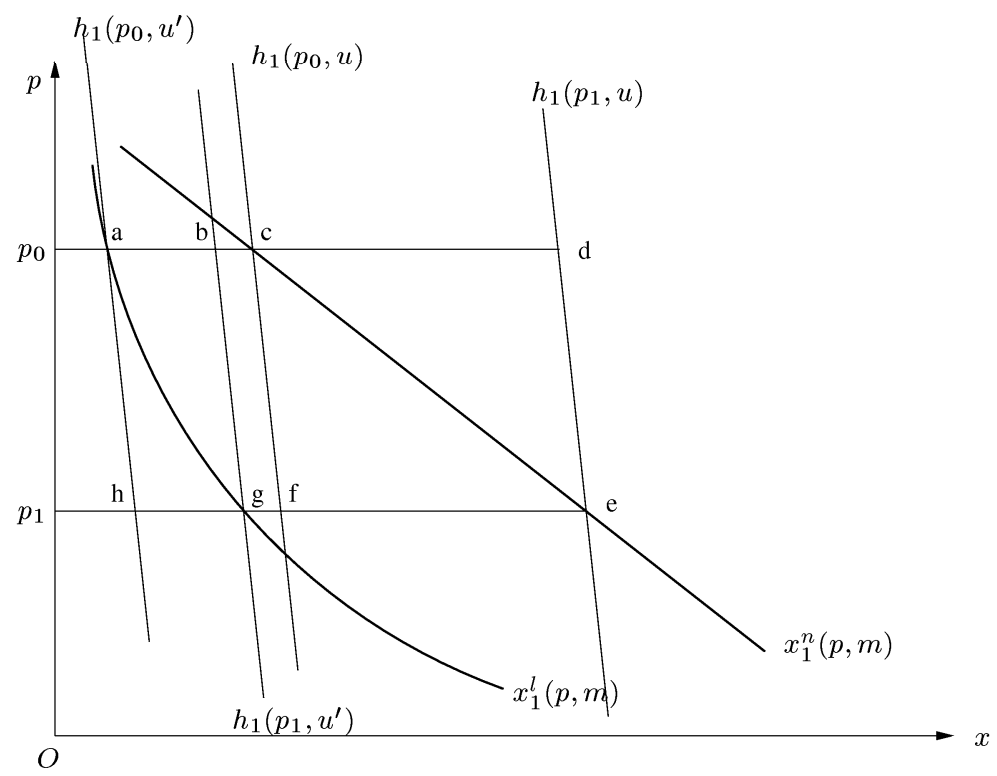

Figure 4: The Effects of a Decrease in $p$

welfare. However, the cause of the bias differs. In the case of non-perishable goods, the fact that a purchase or sale of a good in the current period commits the agent to future consumption levels generates a commitment cost which causes a divergence between the willingness to pay for a good, the willingness to sell, and the respective CV/EV. However, in the case of perishable goods, commitment costs do not arise because the current decisions do not have long-run consequences. Then learning about $\theta$ will not significantly alter the divergence between WTP and WTA. Rather, differences between the reported willingness to pay and the true willingness to pay arise from income reallocation between the two periods.

\section{Discussion and Conclusions}

In this paper, we have presented two simple models of dynamic consumer behavior and considered the implications for welfare measurement when agents can delay transactions while obtaining additional information. In the first model, the consumer is assumed to face the decision of purchasing or selling a good that is non-perishable, implying that the level of consumption of the 
good chosen in the current period will be consumed in the future periods. In this setting, the well understood equivalence between the static Hicksian welfare measures of CV and EV and their behavioral counterparts, WTP and WTA, no longer holds. These results have important implications for understanding the presence of a divergence between WTP and WTA as well as when the divergence should disappear or be small.

In the second model, we study a situation in which the good is perishable, implying that the quantity of consumption can vary freely in each period. Even in this case we find that the availability of information at the time that the consumption decision must be made has important implications for welfare measurement. Fundamentally, when the agent must make a decision today and she knows that additional information will be available later, she may change her income allocation to take advantage of the future information. This will alter her reported welfare values and projected demand.

In both cases, the information available to respondents at the time they answer a stated preference question relative to the information they will ultimately obtain about the good is key. To the extent that there is a difference in the information sets at these different times, there is the potential for erroneous welfare assessment. This finding has implications for researchers designing and analyzing data from stated preference surveys. Specifically, researchers need to be aware of whether the requirements of the survey instrument might generate commitment costs or generate information-restricted demands.

Finally, it is important to note that the theoretical possibility of these information effects does not imply that they will be of significant magnitude in any particular case to warrant concern. Further empirical investigation is needed to understand the circumstances under which these magnitudes are likely to be large and therefore of practical concern. 


\section{References}

Arrow, Kenneth J., and Anthony C. Fisher. 1974. “Environmental Preservation, U ncertainty, and I rreversibility." Q uarterly Journal of Economics 88: 312-19.

D ixit, Avinash K., and Robert S. Pindyck. 1994. Investment U nder U ncertainty. Princeton, N J:

Princeton U niversity Press.

H anemann, W. M ichael. 1989. "Information and the Concept of O ption Value." Journal of Environmental Economics and M anagement 16: 23-37.

. 1991. "W illingness to Pay and W illingness to Accept: H ow M uch C an They D iffer?"

American Economic Review 81: 635-47.

Randall, Alan, and J ohn R. Stoll. 1980. "C onsumer's Surplus in Commodity Space." American Economic Review 70: 449-55.

Zhao, Jinhua, and Catherine L. Kling. 2000. "W illingness-to-Pay, Compensating V ariation, and the Cost of Commitment." W orking Paper, I owa State U niversity. . 2001. "A N ew Explanation for the W T P/W T A D isparity." Economics L etters 73: 293-300. 